\title{
UNICITY THEOREMS FOR THE GAUSS MAPS OF COMPLETE MINIMAL SURFACES, II
}

\author{
Dedicated to Professor Mitsuru Nakai on his 60th birthday
}

\author{
By Hirotaka Fujimoto
}

\section{$\S 1$. Introduction.}

The Gauss map of complete minimal surfaces in $\boldsymbol{R}^{m}$ have many properties which have analogies to value-distribution-theoretic properties of holomorphic curves in the complex projective space. The author gave some of them in his papers [5] [8]. Moreover, in [9] he obtained the following analogy of Nevanlinna's unicity theorem ([11]):

THEOREM. Let $M$ and $\tilde{M}$ be two nonflat minimal surfaces immersed in $\boldsymbol{R}^{3}$ and let $G: M \rightarrow S^{2}$ and $\tilde{G}: \tilde{M} \rightarrow S^{2}$ be the Gauss maps of $M$ and $\tilde{M}$ respectively. Suppose that there is a conformal diffeomorphism $\Phi$ between $M$ and $\tilde{M}$. If $M$ or $\tilde{M}$ is complete and there are seven distinct directions $n_{1}, \cdots, n_{7} \in S^{2}$ such that $G^{-1}\left(n_{j}\right)=(\tilde{G} \cdot \Phi)^{-1}\left(n_{\jmath}\right)(1 \leqq \jmath \leqq 7)$, then $G \equiv \tilde{G} \cdot \Phi$.

He gave also more precise results for the case where both of $M$ and $\tilde{M}$ are complete and have finite total curvature. The purpose of this paper is to give some generalizations of these results to minimal surfaces in $\boldsymbol{R}^{m}$ for the case $m>3$.

As is well-known, the set of all oriented 2-planes in $\boldsymbol{R}^{m}$ containing the origin is canonically identified with the quadric

$$
Q_{m-2}(\boldsymbol{C}):=\left\{\left(w_{1}: \cdots: w_{m}\right) ; w_{1}^{2}+\cdots+w_{m}^{2}=0\right\}
$$

in $P^{m-1}(\boldsymbol{C})$. For a minimal surface $x:=\left(x_{1}, \cdots, x_{m}\right): M \rightarrow \boldsymbol{R}^{m}$ immersed in $\boldsymbol{R}^{m}$ the Gauss map $G$ of $M$ is defined as the map of $M$ into $Q_{m-2}(C)$ which maps each $p \in M$ to the point in $Q_{m-2}(C)$ corresponding to the oriented tangent plane of $M$ at $p$. We may regard $M$ as a Riemann surface with a conformal metric and $G$ as a holomorphic map of $M$ into $P^{m-1}(C)$.

As in the case $m=3$, we consider two nonflat minimal surfaces

$$
x:=\left(x_{1}, \cdots, x_{m}\right): M \rightarrow \boldsymbol{R}^{m}, \quad \tilde{x}:=\left(\tilde{x}_{1}, \cdots, \tilde{x}_{m}\right): \tilde{M} \rightarrow \boldsymbol{R}^{m}
$$

and their Gauss maps $G: M \rightarrow P^{N}(\boldsymbol{C})$ and $\tilde{G}: \tilde{M} \rightarrow P^{N}(\boldsymbol{C})$, where $N:=m-1$. Sup-

Received June 14, 1993. 
pose that there is a conformal diffeomorphism $\Phi$ between $M$ and $\tilde{M}$. Then the Gauss map of the minimal surface $\tilde{x} \cdot \Phi: M \rightarrow \boldsymbol{R}^{m}$ is given by $\tilde{G} \cdot \Phi$. Consider the holomorphic maps $f:=G: M \rightarrow P^{N}(\boldsymbol{C}), g:=\tilde{G} \cdot \Phi: M \rightarrow P^{N}(\boldsymbol{C})$ and assume that they satisfy the following:

Assumption 1.1. There exist hyperplanes $H_{1}, \cdots, H_{q}$ in $P^{N}(\boldsymbol{C})$ located in general position such that

(i) $f^{-1}\left(H_{j}\right)=g^{-1}\left(H_{j}\right) \neq M$ for every $j$,

(ii) $f=g$ on $\bigcup_{j=1}^{q} f^{-1}\left(H_{j}\right)-K$ for a compact subset $K$ of $M$.

The main result is stated as follows:

THEOREM 1.2. Under the above assumption, we have necessarily $f \equiv g$

(A) if $q>m^{2}+m(m-1) / 2$ for the case where $M$ is complete and has infinite total curvature or

(B) if $q \geqq m^{2}+m(m-1) / 2$ for the case where $K=\varnothing$ and $M$ and $\tilde{M}$ are both complete and have finite total curvature.

We shall give some estimates for divisors on an open Riemann surface in $\S 2$ and construct a pseudo-metric with strictly negative curvature associated with two holomorphic maps $f$ and $g$ into $P^{N}(\boldsymbol{C})$ satisfying Assumption 1.1 in $\S 3$. After these preparations, in $\S \S 4 \sim 5$ we shall prove some unicity theorems for holomorphic maps into $P^{N}(\boldsymbol{C})$ defined on an open Riemann surfaces with complete conformal metrics. Theorem 1.2 will be proved in $\S 6$.

\section{§. Some estimates for divisors.}

Let $M$ be a Riemann surface. In this paper, a divisor $\nu$ on $M$ means a map $\nu: M \rightarrow R$ whose support $\operatorname{Supp}(\nu):=\overline{\{z ; \nu(z) \neq 0\}}$ has no accumulation point in $M$. We say that a complex-valued function $u$ on $M$ has mild singularities if it can be written as

$$
|u(z)|=|z-a|^{\sigma} u^{*}(z) \prod_{i}\left|\frac{1}{\log \left|g_{i}(z) v_{i}(z)\right|}\right|^{\tau_{i}}
$$

on a neighborhood of each $a € M$ with a real number $\sigma$, finitely many nonnegative numbers $\tau_{\imath}$, positive $C^{\infty}$ function $u^{*}, v_{i}$ and nonzero holomorphic functions $g_{\imath}$, where $z$ is a holomorphic local coordinate around $a$. For a function $u$ with mild singularities, we define the divisor $\nu_{u}$ of $u$ by

$$
\nu_{u}(a):=\text { the number } \sigma \text { for the representation (2.1) }
$$

for each $a \sqsubseteq M$.

Let $f: M \rightarrow \boldsymbol{P}^{n}(\boldsymbol{C})$ be a nondegenerate holomorphic map. For $a \in M$ taking an open neighborhood $D$ of $a$ contained in the domain of a holomorphic local coordinate, we choose a reduced representation $f:=\left(f_{0}: \cdots: f_{n}\right)$ on $D$, where 
$f_{\imath}$ 's are holomorphic functions on $D$ without common zero. Consider the holomorphic map

$$
F_{k}:=F^{(0)} \wedge F^{(1)} \wedge \cdots \wedge F^{(k)}: D \rightarrow{ }^{k+1} C^{n+1}
$$

for $0 \leqq k \leqq n$, where $F^{(0)} \equiv F:=\left(f_{0}, \cdots, f_{n}\right)$ and $F^{(l)}:=\left(f_{0}^{(l)}, \cdots, f_{n}^{(l)}\right)$ for each $l=0,1, \cdots$. The norm of $F_{k}$ is given by

$$
\left|F_{k}\right|:=\left(\sum_{0 \leqq \imath_{0}<\cdots<i_{k} \leqq n}\left|W\left(f_{\imath_{0}}, \cdots, f_{\imath_{k}}\right)\right|^{2}\right)^{1 / 2},
$$

where $W\left(f_{\imath_{0}}, \cdots, f_{\imath_{k}}\right)$ denotes the Wronskian of $f_{\imath_{0}}, \cdots, f_{\imath_{k}}$. Set $\nu_{k}:=\nu_{\mid F_{k}}$ | for $0 \leqq k \leqq n$. The divisor $\nu_{n}$ is nothing but the divisor of $W\left(f_{0}, f_{1}, \cdots, f_{n}\right)$. These are globally well-defined on $M$. Because, for another reduced representation $f:=\left(\tilde{f}_{0}: \cdots: \tilde{f}_{n}\right)$ we can write $\left(\tilde{f}_{0}, \cdots, \tilde{f}_{n}\right)=h F$ with a nowhere vanishing holomorphic function $h$ and $F_{k}$ is multiplied by $h^{k+1}$, and for another holomorphic local coordinate $\zeta, F_{k}$ is multiplied by $(d z / d \zeta)^{k(k+1) / 2}$.

We now take a hyperplane $H$ with $f(M) \nsubseteq H$ given by

$$
H: \bar{c}_{0} w_{0}+\cdots+\bar{c}_{n} w_{n}=0 .
$$

For each reduced representation $f:=\left(f_{0}: \cdots: f_{n}\right)$ we set

$$
F \equiv F(H):=\bar{c}_{0} f_{0}+\cdots+\bar{c}_{n} f_{n}
$$

and aefine the pull-back of the divisor $H$ via $f$ by $\nu(f, H):=\nu_{F}$, which is welldefined on $M$.

We next consider $q$ hyperplanes $H_{1}, \cdots, H_{q}$ in $P^{n}(\boldsymbol{C})$ given by

$$
H_{\jmath}:\left\langle w, A_{\jmath}\right\rangle \equiv \bar{c}_{j_{0}} w_{0}+\cdots+\bar{c}_{{ }_{j}} w_{n}=0 \quad(1 \leqq j \leqq q),
$$

where $A_{\jmath}:=\left(c_{j 0}, \cdots, c_{\jmath n}\right) \in C^{n+1}-\{0\}$. For $R \subseteq Q:=\{1,2, \cdots, q\}$ we denote by $d(R)$ the dimension of the vector subspace of $\boldsymbol{C}^{n+1}$ generated by $\left\{A_{j} ; \jmath \in R\right\}$. Following [3], we say that $H_{1}, \cdots, H_{q}$ are in $N$-subgeneral position if $d(R)=$ $n+1$ for all $R \subseteq Q$ with $\# R \geqq N+1$, where $\# A$ means the number of elements of a set $A$. In particular case $N=n$, these are said to be in general position. In $[12]$ E. I. Noachka gave the following theorem:

Theorem 2.4. For given hyperplanes $H_{1}, H_{2}, \cdots, H_{q}$ in $P^{n}(\boldsymbol{C})$ located in $N$ subgeneral position, there are some rational numbers $\omega(1), \cdots, \omega(q)$ and $\theta$ satisfying the following conditions;

(i) $0<\omega(j) \leqq \theta \leqq 1(1 \leqq j \leqq q)$,

(ii) $\sum_{j=1}^{q} \omega(j)=n+1+\theta(q-2 N+n-1)$,

(iii) $\frac{n+1}{2 N-n+1} \leqq \theta \leqq \frac{n+1}{N+1}$,

(iv) if $R \subset Q$ and $0<\# R \leqq N+1$, then $\sum_{j \in R} \omega(j) \leqq d(R)$. 
For the proof, see [3] or $[10, \S 2.4]$.

We call constants $\omega(j)(1 \leqq j \leqq q)$ and $\theta$ with the properties of Theorem 2.4 Nochka weights and a Nochka constant for $H_{1}, \cdots, H_{q}$ respectively.

Related to Nochka weights, we have the following:

Proposition 2.5. Let $H_{1}, \cdots, H_{q}$ be hyperplanes in $P^{n}(\boldsymbol{C})$ located in $N$ subgeneral position and let $\omega(1), \cdots, \omega(q)$ be Nochka weights for them, where $q>$ $2 N-n+1$. For each $R \leqq Q:=\{1,2, \cdots, q\}$ with $0<\# R \leqq N+1$ and real constants $E_{1}, \cdots, E_{q}$ with $E_{\jmath} \geqq 1$, there are some $R^{\prime} \subseteq R$ such that $\# R^{\prime}=d\left(R^{\prime}\right)=d(R)$ and

$$
\prod_{\jmath \in R} E_{\jmath}^{\omega(j)} \leqq \prod_{j \in R^{\prime}} E_{\jmath}
$$

For the proof, see [3] or [10, Proposition 2.4.15].

For later use, we shall give the following estimate for divisors.

Proposition 2.6. Let $f$ be a nondegenerate holomorphic map of a domain in $\boldsymbol{C}$ into $P^{n}(\boldsymbol{C})$ with a reduced representation $f=\left(f_{0}: \cdots: f_{n}\right)$ and let $H_{1}, \cdots, H_{q}$ be hyperplanes in $N$-subgeneral position with Nochka weights $\omega(1), \cdots, \omega(q)$ respectively. Then,

$$
\sum_{j=1}^{q} \omega(j) \nu\left(f, H_{j}\right) \leqq \nu_{n}+\frac{n(n+1)}{2}
$$

Proof. For an arbitrary $a \in M$ set $m_{\jmath}:=\nu\left(f, H_{j}\right)(a)$ and $S:=\left\{j ; m_{,}>0\right\}$. Then $\# S \leqq N$. For, otherwise, $f_{\imath}(0 \leqq i \leqq n)$ are represented as linear combinations of $\left\{F\left(H_{j}\right) ; j \in S\right\}$ and so $f_{0}, \cdots, f_{n}$ have a common zero at $a$. We choose a set $R$ with $S \subseteq R \subseteq\{1, \cdots, q\}$ and $\# R=N+1$. Then we see $d(R)=n+1$ by the assumption. Set $E_{\jmath}:=e^{m_{\jmath}}$. By Proposition 2.5 there exists some $j_{0}, j_{1}, \cdots, j_{n}$ in $R$ such that $H_{\jmath_{0}}, \cdots, H_{\jmath_{n}}$ are linearly independent and $\Pi_{j \in R} E_{\jmath}^{\omega(j)} \leqq \Pi_{l=0}^{n} E_{\jmath_{l}}$, so that

$$
\sum_{\jmath \in R} \omega(j) m_{j} \leqq \sum_{l=0}^{n} m_{\jmath_{l}}
$$

Set $\phi_{l}:=F\left(H_{j_{l}}\right)$ and define $\varphi:=\frac{W\left(\psi_{0}, \phi_{1}, \cdots, \psi_{n}\right)}{\psi_{0} \psi_{1} \cdots \psi_{n}}$. Since $f_{0}, f_{1}, \cdots, f_{n}$ are represented as linear combinations of $\phi_{0}, \cdots, \phi_{n}, W\left(f_{0}, f_{1}, \cdots, f_{n}\right)$ is a nonzero constant multiple of $W\left(\psi_{0}, \phi_{1}, \cdots, \phi_{n}\right)$. This implies that

$$
\nu_{\varphi}(a)=\nu_{n}(a)-\sum_{l=0}^{n} m_{\jmath_{l}} .
$$

On the other hand, the meromorphic function $\varphi$ is expanded as 


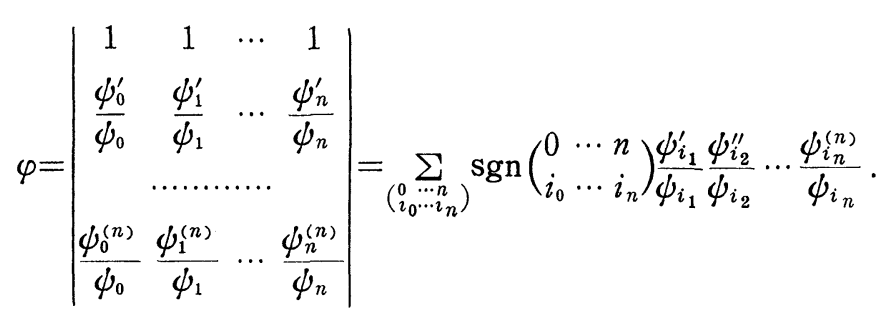

Since the order of the pole of each $\phi_{j}^{(l)} / \phi_{j}$ is at most $l$, each term in the above expansion of $\varphi$ has a pole of order at most $n(n+1) / 2$ at $a$. Therefore, we have $\nu_{\varphi}(a) \geqq-n(n+1) / 2$. By the use of $(2.7)$ and (2.8) we conclude

$$
\sum_{j=1}^{q} \omega(j) \nu\left(f, H_{\jmath}\right)(a) \leqq \sum_{l=0}^{n} m_{\jmath_{l}}=\nu_{n}-\nu_{\varphi}(a) \leqq \nu_{n}+\frac{n(n+1)}{2} .
$$

The proof of Proposition 2.6 is completed.

\section{§3. Pseudo-metrics with strictly negative curvature.}

Let $M$ be an open Riemann surface and $d s^{2}$ a pseudo-metric on $M$, namely, a metric on $M$ with isolated singularities which is locally written as $d s^{2}=$ $\lambda^{2}|d z|^{2}$ in terms of a nonnegative real-valued function $\lambda$ with mild singularities and a holomorphic local coordinate $z$. We define the divisor of $d s^{2}$ by $\nu_{d s}:=\nu_{\lambda}$ for each local expression $d s^{2}=\lambda^{2}|d z|^{2}$, which is globally well-defined on $M$. We say that $d s^{2}$ is a continuous pseudo-metric if $\nu_{d s} \geqq 0$ everywhere.

Definition 3.1. We define the Ricci form of $d s^{2}$ by

$$
\operatorname{Ric}\left[d s^{2}\right]:=-d d^{c}\left[\log \lambda^{2}\right]
$$

for each local expression $\nu_{d s}:=\nu_{\lambda}$, where we mean by $[u]$ the current associated with a locally integrable function $u$ and by $d$ and $d^{c}(:=(\sqrt{-1} / 4 \pi)(\bar{o}-\partial))$ the differential operators for the currents.

Let $f, g$ be distinct nonconstant holomorphic maps of $M$ into $P^{N}(\boldsymbol{C})$ and consider a nonzero function $\chi(w, \tilde{w})$ on $\boldsymbol{C}^{N+1} \times \boldsymbol{C}^{N+1}$ having the following:

PROPERTY 3.2. It is bilinear with respect to the varıables $w$ and $\tilde{w}$, and $\chi(w, w)=0$ for all $w \boxminus \boldsymbol{C}^{N+1}$.

Suppose that they satisfy the following:

Assumption 3.3. There exist hyperplanes $H_{1}, \cdots, H_{q}$ in $P^{N}(C)$ located in general positien such that

(i) $\chi(f, g) \not 0$ and $f^{-1}\left(H_{j}\right)=g^{-1}\left(H_{\jmath}\right) \neq M$ for every $\jmath$,

(ii) $\chi(f, g)=0$ on $\bigcup_{j=1}^{q} f^{-1}\left(H_{\jmath}\right)-K$ for a compact subset $K$ of $M$. 
Take the smallest projective linear subspaces $P_{f}$ and $P_{g}$ of $P^{N}(C)$ which include the images $f(M)$ and $g(M)$ respectively. Set $n_{f}:=\operatorname{dim} P_{f}$ and $n_{g}:=$ $\operatorname{dim} P_{g}$. Then the maps $f: M \rightarrow P_{f}$ and $g: M \rightarrow P_{g}$ are nondegenerate. We regard hyperplanes $H_{1} \cap P_{f}, \cdots, H_{q} \cap P_{f}$ as hyperplanes in $P_{f}$, which are located in $N$-subgeneral position. We take Nochka weights $\omega_{f}(1), \cdots, \omega_{f}(q)$ and a Nochka constant $\theta_{f}$ for these hyperplanes. Similarly, we take $\omega_{g}(1), \cdots, \omega_{g}(q)$ and $\theta_{g}$ for the hyperplanes $H_{1} \cap P_{g}, \cdots, H_{q} \cap P_{g}$ with respect to the map $g$. Choose reduced representations $f:=\left(f_{0}: \cdots: f_{n_{f}}\right), g:=\left(g_{0}: \cdots: g_{n_{g}}\right)$ on $M$ in terms of some homogeneous coordinates on $P_{f}$ and $P_{g}$ respectively. Set $F:=$ $\left(f_{0}, \cdots, f_{n_{f}}\right)$ and $G:=\left(g_{0}, \cdots, g_{n_{g}}\right)$ and consider the maps $F_{k}$ and $G_{k}$ defined as (2.2) for $f$ and $g$. Taking unit vectors $A_{j} \subseteq \boldsymbol{C}^{n_{f}+1}, B_{j} \equiv \boldsymbol{C}^{n^{+1}}$ with

$$
H_{j} \cap P_{f}:\left\langle w, A_{\jmath}\right\rangle=0\left(w \in P_{f}\right), \quad H_{j} \cap P_{g}:\left\langle\tilde{w}, B_{\jmath}\right\rangle=0\left(\tilde{w} \in P_{g}\right),
$$

we define $F\left(H_{j}\right):=\left\langle F, A_{\jmath}\right\rangle, G\left(H_{j}\right):=\left\langle G, B_{\jmath}\right\rangle$ and the contact functions $\varphi_{k}^{f}\left(H_{\jmath}\right)$ : $=\left|F_{k} \vee A_{j}\right|^{2} /\left|F_{k}\right|^{2}$ for $0 \leqq k \leqq n_{f}$ and $\varphi_{k}^{g}\left(H_{j}\right):=\left|G_{k} \vee A_{j}\right|^{2} /\left|G_{k}\right|^{2}$ for $0 \leqq k \leqq n_{g}$, where $X \vee Y$ denotes the interior product of vectors $Y$ and $Y$ (c.f., $[8, \S 3]$ ).

Set $\sigma_{n}:=n(n+1) / 2, \tau_{n}:=\sum_{k=1}^{n} \sigma_{k}$ and

$$
\gamma_{f}:=\theta_{f}\left(q-2 N+n_{f}-1\right), \quad \gamma_{g}:=\theta_{g}\left(q-2 N+n_{g}-1\right) .
$$

Suppose that

$$
\frac{\sigma_{n_{f}}}{\gamma_{f}}+\frac{\sigma_{n_{g}}}{\gamma_{g}}<1
$$

Choose positive numbers $\varepsilon_{1}$, $\varepsilon_{2}$ such that $\gamma_{f}>\varepsilon_{1} \tau_{n_{f}}, \gamma_{g}>\varepsilon_{2} \tau_{n_{g}}$ and

$$
\frac{\sigma_{n f}+\varepsilon_{1} \sigma_{n f+1}}{\gamma_{f}-\varepsilon_{1} \tau_{n_{f}}}+\frac{\sigma_{n_{g}}+\varepsilon_{2} \sigma_{n_{g}+1}}{\gamma_{g}-\varepsilon_{2} \tau_{n_{g}}}<1
$$

and define the functions

$$
\begin{aligned}
& \eta_{f}:=\left(\frac{|F|^{\gamma_{f}-\varepsilon_{1} \sigma_{n}} f^{+1}\left|F_{n_{f}}\right| \prod_{k=0}^{n_{f}}\left|F_{k}\right|^{\varepsilon_{1}}}{\prod_{j=1}^{q}\left(\left|F\left(H_{j}\right)\right| \prod_{k=0}^{n_{f}-1} \log \left(\delta / \varphi_{k}^{f}\left(H_{j}\right)\right)\right)^{\omega_{f}^{(j)}}}\right)^{1 /\left(\sigma_{n_{f}+\varepsilon_{1} \tau_{n}}{ }^{\prime}\right.}, \\
& \eta_{g}:=\left(\frac{\left.|G|^{\gamma_{g}-\varepsilon_{2} \sigma_{n}+1}\left|G_{n}\right| \prod_{k=0}^{n} \mid G_{k}\right]^{\varepsilon_{2}}}{\prod_{j=1}^{q}\left(\left|G\left(H_{j}\right)\right| \Pi_{k=0}^{n} g^{-1} \log \left(\delta / \varphi_{k}^{g}\left(H_{j}\right)\right)\right)^{\omega} g^{(j)}}\right)^{1 /\left(\sigma_{n}+\varepsilon_{2} \tau_{g}{ }^{\prime}\right)},
\end{aligned}
$$

where $\delta$ is a sufficiently large positive constant which is specified later. As is easily seen, $\eta_{f}^{2}|d z|^{2}$ and $\eta_{g}^{2}|d z|^{2}$ are globally well-defined on $M$. Take $p_{1}$ and $p_{2}$ with

$$
p_{1}+p_{2}=1, \quad p_{1} \geqq \frac{\sigma_{n_{f}}+\varepsilon_{1} \tau_{n_{f}}}{\gamma_{f}-\varepsilon_{1} \sigma_{n_{f+1}}}, \quad p_{2} \geqq \frac{\sigma_{n_{g}}+\varepsilon_{2} \tau_{n_{g}}}{\gamma_{g}-\varepsilon_{2} \sigma_{n_{g}+1}}
$$

and define

$$
d \tau^{2}:=\frac{|\chi(F, G)|^{2}}{|F|^{2}|G|^{2}} \eta_{f}^{2 p_{1}} \eta_{g}^{2 p_{2}}|d z|^{2}
$$


Definition 3.9. We say that a continuous pseudo-metric $d s^{2}$ has strictly negative curvature on $M$ if there is a positive constant $C$ such that

$$
-\operatorname{Ric}\left[d s^{2}\right] \geqq C \Omega_{d s^{2}},
$$

where $\Omega_{d s^{2}}$ denotes the area form for $d s^{2}$, namely, $\Omega_{d s^{2}}:=\lambda^{2}(\sqrt{-1} / 2) d z \wedge d \bar{z}$ for each local expression $d s^{2}=\lambda^{2}|d z|^{2}$.

Proposition 3.10. For a sufficiently large $\delta$, the pseudo-metric $d \tau^{2}$ given by (3.8) is continuous and has strictly negative curvature on $M-K$.

Proof. We first show that $\nu_{d \tau}(z) \geqq 0$ for all $z \leqq M$. This is obvious if $z \notin$ $\bigcup_{j=1}^{q} f^{-1}\left(H_{\jmath}\right)\left(=\bigcup_{j=1}^{q} g^{-1}\left(H_{\jmath}\right)\right)$. Otherwise, since

$$
\nu_{n_{f}}-\sum_{j=1}^{q} \omega_{f}(j) \nu\left(f, H_{j}\right) \geqq-\sigma_{n_{f}}, \quad \nu_{n_{g}}-\sum_{j=1}^{q} \omega_{g}(j) \nu\left(g, H_{j}\right) \geqq-\sigma_{n_{g}}
$$

by Proposition 2.6, we obtain

$$
\begin{aligned}
& \nu_{d \tau} \geqq \nu_{\chi}+p_{1}\left(\nu_{n_{f}}-\sum_{j=1}^{q} \omega_{f}(j) \nu\left(f, H_{j}\right)\right) \frac{1}{\sigma_{n_{f}}+\varepsilon_{1} \tau_{n_{f}}} \\
&+p_{2}\left(\nu_{n_{g}}-\sum_{j=1}^{q} \omega_{\boldsymbol{g}}(j) \nu\left(g, H_{j}\right)\right) \frac{1}{\sigma_{n_{g}}+\varepsilon_{2} \tau_{n_{g}}} \\
& \geqq \nu_{\chi}-\frac{p_{1} \sigma_{n_{f}}}{\sigma_{n_{f}}+\varepsilon_{1} \tau_{n_{f}}}-\frac{p_{2} \sigma_{n_{g}}}{\sigma_{n_{g}}+\varepsilon_{2} \tau_{n_{g}}} \\
& \geqq \nu_{\chi}-1 .
\end{aligned}
$$

On the other hand, by the assumption we have $\chi=0$ on $\bigcup_{j=1}^{q} f^{-1}\left(H_{\jmath}\right)$ and hence $\nu_{\chi} \geqq 1$ there. This concludes that $d \tau^{2}$ is continuous on $M$.

To complete the proof, we choose a sufficiently large $\delta$ so that

$$
\begin{aligned}
& d d^{c} \log \eta_{f}^{2} \geqq \frac{\gamma_{f}-\varepsilon_{1} \sigma_{n_{f+1}}}{\sigma_{n_{f}}+\varepsilon_{1} \tau_{n_{f}}} \Omega_{f}+C_{1} \eta_{f}^{2} d d^{c}|z|^{2}, \\
& d d^{c} \log \eta_{g}^{2} \geqq \frac{\gamma_{g}-\varepsilon_{2} \sigma_{n_{g}+1}}{\sigma_{n_{g}}+\varepsilon_{2} \tau_{n_{g}}} \Omega_{g}+C_{1} \eta_{g}^{2} d d^{c}|z|^{2}
\end{aligned}
$$

on $M-\left(K \bigcup_{j=1}^{q} f^{-1}\left(H_{\jmath}\right)\right)$ for some positive constants $C_{1}$ by the same arguments as in [8, pp. 31 32], where $\Omega_{f}:=d d^{c} \log |F|^{2}$ and $\Omega_{g}:=d d^{c} \log |G|^{2}$. We then have

$$
\begin{aligned}
-\operatorname{Ric}\left[d \tau^{2}\right]= & d d^{c} \log |\chi|^{2}-\Omega_{f}-\Omega_{g}+p_{1} d d^{c} \log \eta_{f}^{2}+p_{2} d d^{c} \log \eta_{g}^{2} \\
\geqq & C_{1}\left(p_{1} \eta_{f}^{2}+p_{2} \eta_{g}^{2}\right) d d^{c}|z|^{2} \\
& +\left(p_{1} \frac{\gamma_{f}-\varepsilon_{1} \sigma_{n_{f+1}}}{\sigma_{n_{f}}+\varepsilon_{1} \tau_{n_{f}}}-1\right) \Omega_{f}+\left(p_{2} \frac{\gamma_{g}-\varepsilon_{2} \sigma_{n_{g}+1}}{\sigma_{n_{g}}+\varepsilon_{2} \tau_{n_{g}}}-1\right) \Omega_{g} \\
\geqq & C_{1} \eta_{f}^{2{ }^{p_{1}} \eta_{g}^{2} p_{2}} d d^{c}|z|^{2} .
\end{aligned}
$$


Since we have $|\chi| \leqq C_{2}|F||G|$ for some constant $C_{2}>0$, we can conclude that $d \tau^{2}$ has strictly negative curvature. The proof of Proposition 3.10 is completed.

\section{§4. Unicity theorems for holomorphic curves.}

Let $M$ be an open Riemann surface and $d s^{2}$ a complete conformal metric on $M$. We now recall the following definition and result given in [8].

Definition 4.1. Let $\Omega_{1}$ and $\Omega_{2}$ be $C^{\infty}$ differentiable $(1,1)$-currents on $M$. For some $c>0$, by $\Omega_{1} \prec_{c} \Omega_{2}$ we mean that there exist some divisor $\nu$ and a bounded continuous nonnegative function $k$ with mild singularities on $M$ such that $\nu \geqq c$ everywhere on $\operatorname{Supp}(\nu)$ and

$$
\Omega_{1}+[\nu]=\Omega_{2}+d d^{c}\left[\log k^{2}\right],
$$

where $[\nu]$ denotes the current associated with $\nu$. The notation $\Omega_{1}<\Omega_{2}$ means that $\Omega_{1} \prec_{c} \Omega_{2}$ for some $c>0$.

THEOREM 4.2. Let $M$ be an open Riemann surface with a complete conformal metric $d s^{2}$. If there exists a continuous pseudo-metric $d \tau^{2}$ on $M$ whose curvature is stractly negative on $M-K$ for some compact set $K$ such that, for some constant $p$ with $0<p<1$,

$$
-\operatorname{Ric}\left[d s^{2}\right]<_{1-p} p\left(-\operatorname{Ric}\left[d \tau^{2}\right]\right)
$$

on $M-K$, then $M$ is of finite type, namely, biholomorphic with a compact Riemann surface with finitely many points removed.

For the proof, see $[8$, pp. $24 \sim 27]$.

To state the main result of this section, we give the following:

Definition 4.3. Let $f, g: M \rightarrow P^{N}(C)$ be nonconstant holomorphic maps. We say that they satisfy the condition $(C)_{\rho_{1}, \rho_{2}}$ for some $\rho_{1}, \rho_{2}>0$ if

$$
-\operatorname{Ric}\left[d s^{2}\right]<\rho_{1} \Omega_{f}+\rho_{2} \Omega_{g}
$$

on $M-K$ for some compact set $K$.

THEOREM 4.4. Let $M$ be an open Riemann surface with a complete conformal metric $d s^{2}$ and $f, g$ nonconstant holomorphic maps of $M$ into $P^{N}(C)$. Suppose that, for some $\rho_{1}, \rho_{2}>0$,

(i) $M$ is not of finite type,

(ii) $f$ and $g$ satisfy the condition $(C)_{\rho_{1}, \rho_{2}}$,

(iii) $\gamma_{f}, \gamma_{g}>0$ for the numbers $\gamma_{f}, \gamma_{g}$ defined by (3.4) and Assumption 3.3 holds for some $\chi$ with Property 3.2.

Then, it holds that 


$$
\left(\rho_{1}+1\right) \frac{\sigma_{n f}}{\gamma_{f}}+\left(\rho_{2}+1\right) \frac{\sigma_{n g}}{\gamma_{g}} \geqq 1
$$

Proof. The proof is given by reduction to absurdity. Suppose that the conclusion does not hold. We then have the stituation considered in $\S 3$. We use the previous notations without permission. Set

$$
A:=\frac{\gamma_{f}-\varepsilon_{1} \sigma_{n_{f+1}}}{\sigma_{n_{f}}+\varepsilon_{1} \tau_{n_{f}}}, \quad B:=\frac{\gamma_{g}-\varepsilon_{2} \sigma_{n_{g+1}}}{\sigma_{n_{g}}+\varepsilon_{2} \tau_{n_{g}}},
$$

where $\varepsilon_{1}$ and $\varepsilon_{2}$ are chosen so that $\gamma_{f}>\varepsilon_{1} \sigma_{n f+1}>0, \gamma_{g}>\varepsilon_{2} \rho_{n_{g}+1}>0$ and

$$
\frac{\rho_{1}+1}{A}+\frac{\rho_{2}+1}{B}<1 \text {. }
$$

Set

$$
p_{1}:=\frac{B \rho_{1}+\rho_{2}-\rho_{1}}{A \rho_{2}+B \rho_{1}}, \quad p_{2}:=\frac{A \rho_{2}+\rho_{1}-\rho_{2}}{A \rho_{2}+B \rho_{1}} .
$$

Then, we see easily

$$
p_{1} A-1>0, \quad p_{2} B-1>0, \quad p_{1}+p_{2}=1
$$

and

$$
\frac{p_{1} A-1}{\rho_{1}}=\frac{p_{2} B-1}{\rho_{2}}=\frac{A B-A-B}{A \rho_{2}+B \rho_{1}}>1 .
$$

Using these constants $\varepsilon_{1}, \varepsilon_{2}$ and a sufficiently large $\delta$, we define the functions $\eta_{f}$ and $\eta_{g}$ by (3.7) and pseudo-metric $d \tau^{2}$ by (3.8), which is continuous and has strictly negative on $M-K$ according to Proposition 3.10 .

Now, we represent each $H_{\jmath}$ as

$$
H_{j}: \bar{c}_{j 0} w_{0}+\cdots+\bar{c}_{j N} w_{N}=0 \text {. }
$$

As in [8, p. 32], taking some holomorphic local coordinate $z$, for each $j, k$ $\left(1 \leqq j \leqq q, 0 \leqq k \leqq n_{f}-1\right)$ we choose $i_{1}, \cdots, i_{k}$ with $0 \leqq \imath_{1}<\cdots<i_{k} \leqq N$ such that

$$
\phi_{j k}^{2}:=\sum_{l \neq \imath_{1}, \cdots, \imath_{k}} \bar{c}_{j l} W\left(f_{l}, f_{\imath_{1}}, \cdots, f_{\imath_{k}}\right) \neq 0 \text {, }
$$

where we set $\phi_{j_{0}}^{2}:=F\left(H_{j}\right)$. We then have $\left|\psi_{j_{k}}^{2}\right|^{2} /\left|F_{k}\right|^{2} \leqq \varphi_{k}^{f}\left(H_{j}\right)$ and, by the theorem of identity, $\phi_{j_{k}}^{\zeta} \neq 0$ for every holomorphic local coordinate $\zeta$. Set

$$
k_{f}:=\left(\frac{\prod_{1 \leqq j \leq q, 0 \leqq k \leqq n} f_{f^{-1}}\left|\phi_{j_{k}}^{2}\right|^{\varepsilon_{1} / q} \log ^{\omega f_{f}^{(j)}}\left(\delta / \varphi_{k}^{f}\left(H_{j}\right)\right)}{\left.\prod_{0 \leqq k \leqq n}\right)_{f^{-1}}\left|F_{k}\right|^{\varepsilon_{1}}}\right)^{1 /\left(\sigma_{n_{f}+\varepsilon_{1} \tau_{n}}\right)},
$$

which is a well-defined function with mild singularities on $M-K$. Since

$$
\frac{\left|\psi_{j k}^{z}\right|^{\varepsilon_{1} / q} \log ^{\omega_{f}^{(j)}}\left(\delta / \varphi_{k}^{f}\left(H_{j}\right)\right)}{\left|F_{k}\right|^{\varepsilon_{1} / q}} \leqq \sup _{0<x \leqq 1} x^{\varepsilon_{1} / q} \log ^{\omega_{f}(\jmath)}\left(\frac{\delta}{x^{2}}\right)<+\infty,
$$


$k_{f}$ is bounded. Set

$$
\phi_{1}:=\left(\frac{\left|F_{n_{f}}\right| \Pi_{j, k}\left|\phi_{j k}^{z}\right|^{\varepsilon_{1} / q}}{\prod_{\jmath=1}^{q}\left|F\left(H_{f}\right)\right|^{\omega_{f}(\jmath)}}\right)^{1 /\left(\sigma_{n_{f}}+\varepsilon_{1} \tau_{n_{f}}\right)}
$$

on the domain of each holomorphic local coordinate $z$. Then, we have $\nu_{\eta_{f}} \leqq \nu_{\phi_{1}}$, and $\eta_{f} k_{f}=|F|^{A} \phi_{1}$, so that

$$
d d^{c} \log \left(\eta_{f} k_{f}\right)^{2}=A \Omega_{f}+d d^{c} \log \left|\phi_{1}\right|^{2} .
$$

Similarly, we can choose a bounded continuous nonnegative function $k_{g}$ with mild singularities and a locally defined nonzero meromorphic function $\phi_{2}$ satisfying the condition $\nu_{\eta_{g}} \leqq \nu_{\phi_{2}}$ such that

$$
d d^{c} \log \left(\eta_{g} k_{g}\right)^{2}=B \Omega_{g}+d d^{c} \log \left|\phi_{2}\right|^{2} .
$$

On the other hand, we have $d d^{c} \log \left|\phi_{i}\right|^{2}=\left[\nu_{\phi_{l}}\right](i=1,2)$ by Poincaré-Lelong formula. Therefore, we obtain

$$
\begin{aligned}
& - \text { Ric }\left[d \tau^{2}\right]+p_{1} d d^{c} \log k_{f}^{2}+p_{2} d d^{c} \log k_{g}^{2} \\
& \quad=\left[\nu_{x}\right]-\Omega_{f}-\Omega_{g}+p_{1} d d^{c} \log \left(\eta_{f} k_{f}\right)^{2}+p_{2} d d^{c} \log \left(\eta_{g} k_{g}\right)^{2} \\
& \quad=\left(p_{1} A-1\right) \Omega_{f}+\left(p_{2} B-1\right) \Omega_{g}+\left[\nu_{0}\right] .
\end{aligned}
$$

where $\nu_{0}:=\nu_{\chi}+p_{1} \nu_{\phi_{1}}+p_{2} \nu_{\phi_{2}}$. By (4.7) and (4.8) this yields that

$$
-\mathrm{Ric}\left[d \tau^{2}\right]+d d^{2} \log k_{f}^{2 p_{1}} k_{g}^{2 p_{2}}=\frac{A B-A-B}{A \rho_{2}+B \rho_{1}}\left(\rho_{1} \Omega_{f}+\rho_{2} \Omega_{g}\right)+\left[\nu_{0}\right] \text {. }
$$

We have also the inequality $\nu_{0} \geqq \nu_{x}+p_{1} \nu_{n_{f}}+p_{2} \nu_{\eta_{g}}=\nu_{d \tau} \geqq 0$ on $M-K$ and, moreover, there is a positive constant $c_{0}$ with $\iota_{0} \geqq c_{0}$ on $\operatorname{Supp}\left(\boldsymbol{\nu}_{0}\right)$. Set

$$
p:=\frac{A \rho_{2}+B \rho_{1}}{A B-A-B} \text {. }
$$

Then, $0<p<1$ by (4.8) and the identity (4.9) can be rewritten as

$$
\rho_{1} \Omega_{f}+\rho_{2} \Omega_{g} \prec_{p c_{0}} p\left(-\operatorname{Ric}\left[d \tau^{2}\right]\right) .
$$

The assumption (ii) yields that

$$
-\operatorname{Ric}\left[d s^{2}\right]<\rho_{1} \Omega_{f}+\rho_{2} \Omega_{g} \prec p\left(-\operatorname{Ric}\left[d \tau^{2}\right]\right) .
$$

We may write this

$$
-\operatorname{Ric}\left[d s^{2}\right] \prec_{1-p} p\left(-\operatorname{Ric}\left[d \tau^{2}\right]\right),
$$

because we can choose $\varepsilon_{1}$ and $\varepsilon_{2}$ so that $1-p$ is sufficiently small. This contradicts Theorem 4.2. The proof of Theorem 4.4 is completed. 


\section{§. Holomorphic maps defined on a Riemann surface of finite type.}

In this section, we give a unicity theorem similar to the previous section for holomorphic maps into $P^{N}(\boldsymbol{C})$ defined on an open Riemann surface $M$ of finite type which has a complete conformal metric $d s^{2}$.

DEFinition 5.1. Let $f, g: M \rightarrow P^{N}(C)$ be nonconstant holomorphic maps. We say that they satisfy the condition $(C)_{\rho_{1}, \rho_{2}}^{\prime}$ for $\rho_{1}, \rho_{2}>0$ if there is a bounded continuous nonnegative function $k$ with mild singularities such that

$$
-\operatorname{Ric}\left[d s^{2}\right] \leqq \rho_{1} \Omega_{f}+\rho_{2} \Omega_{g}+d d^{c} \log k^{2}
$$

on $M$.

THEOREM 5.3. In the same situation as in Theorem 4.4, suppose that, for some $\rho_{1}, \rho_{2}>0$,

(i) $M$ is of finite type,

(ii) $f$ and $g$ satisfy the condition $(C)_{\rho_{1}, \rho_{2}}^{\prime}$,

(iii) $\gamma_{f}, \gamma_{g}>0$ and Assumption 3.3 holds for $K:=\varnothing$ and some $\chi$ with Property 3.2 .

Then, it holds that

$$
\left(\rho_{1}+1\right) \frac{\sigma_{n}}{\gamma_{f}}+\left(\rho_{2}+1\right) \frac{\sigma_{n_{g}}}{\gamma_{g}} \geqq 1
$$

Proof. Assume that the conclusion does not hold. We use the same notations as in the proof of Theorem 4.4 , where $K:=\varnothing$. Using the same constants $p_{1}, p_{2}$ and functions $\eta_{f}, \eta_{g}$ as in the proof of Theorem 4.4, we construct a continuous pseudo-metric $d \tau^{2}$ on $M$, which has strictly negative curvature on $M$. Here, we note that the universal covering surface of $M$ is biholomorphic with the unit disc. For, there is no continuous pseudo-metric with strictly negative curvature on a Riemann surface whose universal covering surface is biholomorphic with $\boldsymbol{C}$. By the generalized Schwarz' lemma ([1, pp. 12 14]), there exists a positive constant $C_{0}$ such that

$$
d \tau^{2} \leqq C_{0} d \sigma_{M}^{2}
$$

where $d \sigma_{M}^{2}$ denotes the Poincaré metric on $M$. By the assumption, $M$ is biholomorphic with a compact Riemann surface $\bar{M}$ with finitely many points $a_{l}$ 's removed. For each $a_{l}$ we take a neighborhood $U_{l}$ of $a_{l}$ which is biholomorphic with $\Delta^{*}:=\{z ; 0<|z|<1\}$, where $z\left(a_{l}\right)=0$. The Poincaré metric on the domain $\Delta^{*}$ is given by

$$
d \sigma_{\Delta *}:=\frac{4|d z|^{2}}{|z|^{2} \log ^{2}|z|^{2}}
$$

By the use of the distance decreasing property of Poincare metric we have 


$$
d \tau^{2} \leqq C_{l} \frac{|d z|^{2}}{|z|^{2} \log ^{2}|z|^{2}}
$$

for some $C_{l}>0$. This implies that, for a neighborhood $U_{l}^{*}$ of $a_{l}$ which is relatively compact in $U_{l}$,

$$
\int_{U_{l}^{*}} \Omega_{d \tau^{2}}<+\infty
$$

Since $\bar{M}$ is compact, we have

$$
\int_{M} \Omega_{d \tau^{2}} \leqq \int_{\bar{M}-\cup_{l} U_{l}^{*}} \Omega_{d \tau^{2}}+\sum_{l} \int_{U_{l}^{*}} \Omega_{d \tau^{2}}<+\infty
$$

We now take a nowhere zero holomorphic form $\omega$ on $M$. Since $d d^{c} \log \eta_{f}^{2}$ $\geqq A \Omega_{f}$ and $d d^{c} \log \eta_{g}^{2} \geqq B \Omega_{g}$ by (3.11), we can find subharmonic functions $v_{1}$ and $v_{2}$ such that

$$
\eta_{f}^{2}|d z|^{2}=e^{v_{1}}|F|^{2 A}|\omega|^{2}, \quad \eta_{g}^{2}|d z|^{2}=e^{v_{2}}|G|^{2 B}|\omega|^{2} .
$$

Set $v_{3}:=\log |\chi|^{2}+p_{1} v_{1}+p_{2} v_{2}$ and

$$
v:=v_{3}+\left(p_{1} A-1-\rho_{1}\right) \log |F|^{2}+\left(p_{2} B-1-\rho_{2}\right) \log |G|^{2},
$$

which is subharmonic by (4.8). We then have

$$
d \tau^{2}=e^{v_{3}}|F|^{2\left(p_{1} A-1\right)}|G|^{2\left(p_{2} B-1\right)}|\omega|^{2}=e^{v}|F|^{2 \rho_{1}}|G|^{2 \rho_{2}}|\omega|^{2} .
$$

Now, take the functions $\lambda>0$ with $d s^{2}=\lambda^{2}|\omega|^{2}$ and $k$ as in Definition 5.1. By (5.2) there is a subharmonic function $w(\not \equiv-\infty)$ such that

$$
e^{w} \lambda^{2}=k^{2}|F|^{2 \rho_{1}}|G|^{2 \rho_{2}} .
$$

Combining this with (5.5), we obtain

$$
e^{v+w} d s^{2} \leqq C_{0} d \tau^{2}
$$

on $M$ for some positive constant $C_{0}$. Here, we can apply the result of S. T. Yau in [15] to see

$$
\int_{M} e^{v+w} \Omega_{d s^{2}}=+\infty
$$

because $M$ is complete with respect to the metric $d s^{2}$ and $v+w$ is subharmonic. This contradicts the assertion (5.4). The proof of Theorem 5.3 is completed.

Related to Theorem 5.3, we shall prove more precise unicity theorem of holomorphic curve for a particular case. To state this, we give the following:

DEFINITION 5.6. Let $f$ be a nonconstant holomorphic map of an open Riemann surface $M$ into $P^{N}(\boldsymbol{C})$. We say that $f$ has an essential singularity at an 
isolated end of $M$ if we can take a not relatively compact connected open subset $D$ of $M$, called a neighborhood of the end, such that there is a biholomarphic map $\Phi$ of $\{z \in C ; 0<|z|<2\}$ onto an open neighborhood of $\bar{D}$ satisfying the condition that $\Phi(\partial D)=\{z ;|z|=1\}, \Phi(D)=\Delta^{*}:=\{z ; 0<|z|<1\}$ and the map $f \cdot \Phi: \Delta^{*} \rightarrow P^{N}(\boldsymbol{C})$ has an essential singularity at the origin.

THEOREM 5.7. Let $M$ be an open Riemann surface and $f, g$ nonconstant holomorphic maps of $M$ into $P^{N}(C)$ at least one of which has an essential singularity at an isolated end of $M$. Suppose that $\gamma_{f}, \gamma_{g}>0$ and Assumption 3.3 holds for some $\chi$ with Property 3.2 on some neighborhood of the end. Then

$$
\frac{\sigma_{n f}}{\gamma_{f}}+\frac{\sigma_{n_{g}}}{\gamma_{g}} \geqq 1
$$

For the proof, we recall the second main theorem in the classical value distribution theory of holomorphic curves. Let $f$ be a nonconstant holomorphic map of an open neighborhood of $\Delta_{s,+\infty}:=\{z ; s \leqq|z|<+\infty\}$ into $P^{N}(C)$. The order function of $f$ is defined by

$$
T_{f}(r):=\int_{8}^{r} \frac{d t}{t} \int_{s \leqq|z|<t} \Omega_{f},
$$

which can be rewritten as

$$
T_{f}(r)=\frac{1}{2 \pi} \int_{0}^{2 \pi} \log \left|F\left(r e^{i \theta}\right)\right| d \theta-\frac{1}{2 \pi} \int_{0}^{2 \pi} \log \left|F\left(s e^{i \theta}\right)\right| d \theta+O(\log r)
$$

with $F:=\left(f_{0}, \cdots, f_{n}\right)$ for a reduced representation $f:=\left(f_{0}: \cdots: f_{n}\right)([10$, Corol. lary 3.1.12]). For a divisor $\nu$ on an open neighborhood of $\Delta_{s,+\infty}$ the counting function of $\nu$ is defined by

$$
N(r, \nu):=\int_{s}^{r}\left(\sum_{s \leqq|z| \leqq t} \nu(z)\right) \frac{d t}{t} .
$$

Let $H$ be a hyperplane with $f\left(\Delta_{s, \infty}\right) \nsubseteq H$. By definition, the counting function of $H$ for $f$ is given by

$$
N_{f}(r, H):=N(r, \nu(f, H)) .
$$

According to Jensen's formula, we have

$$
N_{f}(r, H)=\frac{1}{2 \pi} \int_{0}^{2 \pi} \log \left|F(H)\left(r e^{i \theta}\right)\right| d \theta-\frac{1}{2 \pi} \int_{0}^{2 \pi} \log \left|F(H)\left(s e^{i \theta}\right)\right| d \theta+O(\log r),
$$

where $F(H)$ is the function defined by (2.3) ([10, Corollary 3.1.8]).

The second main theorem in value distribution theory is stated as follows:

THEOREM 5.9. Let $f: \Delta_{s,+\infty} \rightarrow P^{N}(\boldsymbol{C})$ be a nonconstant holomorphic map and $H_{1}, \cdots, H_{q}$ hyperplanes in $P^{N}(\boldsymbol{C})$ located in general position such that $f\left(\Delta_{s,+\infty}\right) \nsubseteq$ $\cup_{j} H_{j}$. Consider the least projective linear subspace $P_{f}$ which includes the image 
of $f$ and set $n_{f}:=\operatorname{dim} P_{f}$. Take Nochka weights $\omega_{f}(1), \cdots, \omega_{f}(q)$ and a Nochka constant $\theta_{f}$ for $f$ considered as a map into $P_{f}$. Then,

$$
\gamma_{f} T_{f}(r) \leqq \sum_{j=1}^{q} \omega_{f}(j) N_{f}\left(r, H_{j}\right)-N\left(r, \nu_{n_{f}}\right)+O\left(\log \left(r T_{f}(r)\right)\right.
$$

for all $r$ not including in a set $E$ with $\int_{E}(1 / r) d r<\infty$, where $\gamma_{f}=\theta_{f}\left(q-2 N+n_{f}-1\right)$.

This is given in [12]. The details of the proof are described in [2] (cf., [10, Theorem 3.2.12]).

Proof of Theorem 5.7. Changing notation if necessary, we may assume that $f$ has an essential singularity at an isolated end of $M$. Then there is a not relatively compact open subset $D$ of $M$ and a map $\Phi$ satisfying the conditions stated in Definition 5.6. By the identity theorem we have only to prove that $f \cdot \Phi \equiv g \cdot \Phi$ on $\Delta^{*}$. On the other hand, $\Delta^{*}$ is biholomorphically equivalent to $\Delta_{1,+\infty}$. Therefore, there is no harm in assuming that $M=\Delta_{1,+\infty}$ and $f$ has an essential singularity at $\infty$. By Theorem 5.9 we have (5.10) and, similarly,

$$
\gamma_{g} T_{g}(r) \leqq \sum_{j=1}^{q} \omega_{g}(j) N_{g}\left(r, H_{j}\right)-N\left(r, \nu_{n_{g}}\right)+O\left(\log \left(r T_{g}(r)\right)\right.
$$

outside a set $E$ with $\int_{E}(1 / r) d r<\infty$. On the other hand, by Proposition 2.6 we get

$$
\begin{aligned}
& \sum_{j=1}^{q} \omega_{f}(j) N_{f}\left(r, H_{j}\right)-N\left(r, \nu_{n_{f}}\right) \leqq \sigma_{n_{f}} N\left(r, \min \left(\tilde{\nu}_{f}, 1\right)\right), \\
& \sum_{j=1}^{q} \omega_{g}(j) N_{g}\left(r, H_{j}\right)-N\left(r, \nu_{n_{g}}\right) \leqq \sigma_{n_{g}} N\left(r, \min \left(\tilde{\nu}_{g}, 1\right)\right),
\end{aligned}
$$

where $\tilde{\nu}_{f}:=\sum_{j} \nu\left(f, H_{j}\right)$ and $\tilde{\nu}_{g}:=\sum_{j} \nu\left(g, H_{j}\right)$. By Assumption 3.3 we have easily

$$
N\left(r, \min \left(\tilde{\mathcal{\nu}}_{f}, 1\right)\right) \leqq N(r, \chi(f, g)), \quad N\left(r, \min \left(\tilde{\nu}_{g}, 1\right)\right) \leqq N(r, \chi(f, g)) .
$$

By (5.8) and the bilinearity of $\chi$,

$$
N\left(r, \nu_{\chi}(f, g)\right) \leqq T_{f}(r)+T_{g}(r)+O(\log r) .
$$

These imply that

$$
T_{f}(r)+T_{g}(r) \leqq\left(\frac{\sigma_{n f}}{\gamma_{f}}+\frac{\sigma_{n_{g}}}{\gamma_{g}}\right)\left(T_{f}(r)+T_{g}(r)\right)+O\left(\log \left(r T_{f}(r) T_{g}(r)\right)\right) .
$$

On the other hand, since $f$ has an essential singularity at $\infty$, it holds that

$$
\lim _{r \rightarrow \infty} \frac{\log r}{T_{f}(r)+T_{g}(r)}=0
$$


([10, Proposition 3.3.3]). Dividing both sides of (5.11) by $T_{f}(r)+T_{g}(r)$ and tending $r$ to $\infty$, we can conclude that

$$
\frac{\sigma_{n}}{\gamma_{f}}+\frac{\sigma_{n g}}{\gamma_{g}} \geqq 1
$$

This gives Theorem 5.7.

\section{§ 6. Unicity theorems for the Gauss maps of complete minimal surfaces.}

In this section, we shall give some unicity theorems for the Gauss map of complete minimal surfaces in $\boldsymbol{R}^{m}$ by applying the results in the previous sections.

Consider two nonflat minimal surfaces

$$
x:=\left(x_{1}, \cdots, x_{m}\right): M \rightarrow \boldsymbol{R}^{m}, \quad \tilde{x}:=\left(\tilde{x}_{1}, \cdots, \tilde{x}_{m}\right): \tilde{M} \rightarrow \boldsymbol{R}^{m}
$$

and let $G$ and $\tilde{G}$ be the Gauss maps of $M$ and $\tilde{M}$ respectively. Assume that there is a conformal diffeomorphism $\Phi$ of $M$ onto $\tilde{M}$. We may regard $M$ and $\tilde{M}$ as Riemann surfaces and $\Phi$ as a biholomorphic isomorphism between $M$ and $\tilde{M}$. Consider the maps

$$
f:=G: M \rightarrow P^{N}(\boldsymbol{C}), \quad g:=\tilde{G} \cdot \Phi: M \rightarrow P^{N}(\boldsymbol{C}),
$$

where $N:=m-1$, and holomorphic forms $\omega_{i}:=\partial x_{\imath}$ on $M$ and $\tilde{\omega}_{i}:=\partial \tilde{x}_{\imath}$ on $\tilde{M}$. As is well-known, the Gauss maps of $M$ and $\tilde{M}$ are represented as

$$
G=\left(\omega_{1}: \cdots: \omega_{m}\right), \quad \tilde{G}=\left(\tilde{\omega}_{1}: \cdots: \tilde{\omega}_{m}\right),
$$

and the induced metrics of $M$ and $\tilde{M}$ are given by

$$
d s^{2}=2\left(\left|\boldsymbol{\omega}_{1}\right|^{2}+\cdots+\left|\boldsymbol{\omega}_{m}\right|^{2}\right), \quad d \tilde{s}^{2}=2\left(\left|\widetilde{\omega}_{1}\right|^{2}+\cdots+\left|\widetilde{\boldsymbol{\omega}}_{m}\right|^{2}\right)
$$

respectively (e.g., [13]). We can easily obtain the following:

$$
-\operatorname{Ric}\left[d s^{2}\right]=-\operatorname{Ric}\left[\Phi^{*}\left(d \tilde{s}^{2}\right)\right]=\Omega_{f}=\Omega_{g} .
$$

Therefore, $f$ and $g$ satisfy the condition $(C)_{1 / 2,1 / 2}$, where we may take $K=\varnothing$ in Definition 4.3 and hence the condition $(C)_{1 / 2,1 / 2}^{\prime}$ is also satisfied.

THEOREM 6.4. In the above situation, assume that $x: M \rightarrow \boldsymbol{R}^{m}$ is complete and has infinite total curvature and that $f$ and $g$ satisfy Assumption 1.1. If $q>$ $m^{2}+m(m-1) / 2$, then $f \equiv g$.

For a paricular case where $f$ has an essential singularity at an isolated end, the same conclusion holds under the only assumption $q>m^{2}$.

Proof. Assume that $f \not \equiv g$ under the assumption of the first part of Theorem 6.4. For reduced representations $f=\left(f_{1}: \cdots: f_{m}\right)$ and $g=\left(g_{1}: \cdots: g_{m}\right)$, the 
function

$$
\chi\left(\left(w_{1}, \cdots, w_{m}\right) ;\left(\tilde{w}_{1}, \cdots, \tilde{w}_{m}\right)\right):=w_{i} \tilde{w}_{j}-w_{j} \tilde{w}_{\imath}
$$

satisfies Assumption 3.3 for some distinct indices $i, \jmath$. Setting $\rho_{1}=\rho_{2}:=1 / 2$, we apply Theorem 4.4 to see

$$
\frac{n_{f}\left(n_{f}+1\right)}{2 \theta_{f}\left(q-N+n_{f}-1\right)}+\frac{n_{g}\left(n_{g}+1\right)}{2 \theta_{g}\left(q-N+n_{g}-1\right)} \geqq \frac{2}{3} .
$$

On the other hand, by Theorem 2.4 (iii) we see

$$
\frac{n_{f}\left(n_{f}+1\right)}{\theta_{f}\left(q-2 N+n_{f}-1\right)} \leqq \frac{n_{f}\left(2 N-n_{f}+1\right)}{q-2 N+n_{f}-1}
$$

and, since $1 \leqq n_{f} \leqq N$ and $q>(N+1)^{2}$, we have

$$
\begin{aligned}
& \frac{N(N+1)}{q-N-1}-\frac{n_{f}\left(2 N-n_{f}+1\right)}{q-2 N+n_{f}-1} \\
& \quad=\frac{\left(N-n_{f}\right)\left(\left(N-n_{f}+1\right) q-(N+1)\left(2 N-n_{f}+1\right)\right)}{(q-N-1)\left(q-2 N+n_{f}-1\right)} \geqq 0 .
\end{aligned}
$$

These are also true if $\theta_{f}$ and $n_{f}$ are replaced by $\theta_{g}$ and $n_{g}$ respectively. Thus, we obtain

$$
\frac{n_{f}\left(n_{f}+1\right)}{\theta_{f}\left(q-2 N+n_{f}-1\right)} \leqq \frac{N(N+1)}{q-N-1}, \quad \frac{n_{g}\left(n_{g}+1\right)}{\theta_{g}\left(q-2 N+n_{g}-1\right)} \leqq \frac{N(N+1)}{q-N-1} .
$$

and so

$$
\frac{N(N+1)}{q-N-1} \geqq \frac{2}{3}
$$

This leads to an absurd conclusion

$$
q \leqq \frac{3}{2} i N(N+1)+N+1=m^{2}+\frac{m(m-1)}{2} .
$$

The first part of Theorem 6.4 is completely proved.

The proof of the latter half is also given by reduction to absurdity. Suppose that $f \not \equiv g$, and take some $\chi$ with Property 3.2 which satisfies Assumption 3.3. By assumption, $f$ has an essential singularity at an isolated end and $q>(N+1)^{2}$. Then, since $\gamma_{f}, \gamma_{g}>0$ for the constants $\gamma_{f}$ and $\gamma_{g}$ defined by (3.4), we can apply Theorem 5.7 to get

$$
\frac{n_{f}\left(n_{f}+1\right)}{2 \theta_{f}\left(q-N+n_{f}-1\right)}+\frac{n_{g}\left(n_{g}+1\right)}{2 \theta_{g}\left(q-N+n_{g}-1\right)} \geqq 1 .
$$

The assertions (6.5) remains valid in this case too. By (6.6) we conclude

$$
\frac{N(N+1)}{q-N-1} \geqq 1 \text {. }
$$


This contradicts the assumption for $q$. The proof of Theorem 6.4 is completed.

THEOREM 6.7. In the same situation as in Theorem 6.4, suppose that M. $\tilde{M}$ are both complete and have finite total curvature. If $f$ and $g$ satisfy Assumption 1.1 for $K=\varnothing$ and $q \geqq m^{2}+m(m-1) / 2$, then $f \equiv g$.

Proof. Assume that $f \not \equiv g$ and take some $\chi$ with Property 3.2 which satisfies Assumption 3.3. For our purpose, we may replace $\tilde{x}: \tilde{M} \rightarrow \boldsymbol{R}^{m}$ by $\tilde{x} \cdot \Phi: M \rightarrow \boldsymbol{R}^{m}$ and so we may assume that $\tilde{M}=M$ and $\Phi$ is the identity map. By ChernOsserman's theorem ([4]), we may set $M=\bar{M}-\left\{a_{1}, \cdots, a_{K}\right\}$ for a compact Riemann surface $\bar{M}$ and the forms $\omega_{i}:=\partial x_{\imath}, \widetilde{\omega}_{i}:=\partial \tilde{x}_{\imath}(1 \leqq i \leqq m)$ are meromorphically extended to $\bar{M}$. The induced metrics $d s^{2}, d \tilde{s}^{2}$ are also extended to $\bar{M}$ as pseudometrics. We consider the numbers $n_{f}, n_{g}, \omega_{f}(j)$ 's, $\omega_{g}(j)$ 's, $\theta_{f}, \theta_{g}, \gamma_{f}$ and $\gamma_{g}$ defined in $\S 3$. The assertion (6.5) holds in this case too. The assumption implies that

$$
\frac{\sigma_{f}}{\gamma_{f}} \leqq \frac{N(N+1)}{2(q-N-1)} \leqq \frac{1}{3}, \quad \frac{\sigma_{g}}{\gamma_{g}} \leqq \frac{N(N+1)}{2(q-N-1)} \leqq \frac{1}{3} .
$$

Choose sufficiently small positive rational numbers $\varepsilon_{1}$ and $\varepsilon_{2}$ such that the constants $A$ and $B$ defined by (4.5) are both larger than $3-\varepsilon$ for an arbitrarily pre-assigned positive number $\varepsilon$. Settign $p_{1}=p_{2}=1 / 2$, we define the pseudometric $d \tau^{2}$ by (3.8), whose curvature is strictly negative on $M$.

Now, we choose a nonzero vector $\left(c_{1}, \cdots, c_{m}\right)$ such that

$$
\begin{aligned}
& f(M) \cup g(M) \nsubseteq H_{0}:=\left\{\left(w_{1}: \cdots: w_{m}\right) ; \bar{c}_{1} w_{1}+\cdots+\bar{c}_{m} w_{m}=0\right\}, \\
& \omega:=\bar{c}_{1} \omega_{1}+\cdots+\bar{c}_{m} \omega_{m} \not \equiv 0, \\
& \left.\nu_{\omega}\left(a_{l}\right)=\nu_{d s}\left(a_{l}\right)\right), \quad \nu_{\omega}\left(a_{l}\right)=\nu_{d \tilde{s}}\left(a_{l}\right) \quad(1 \leqq l \leqq K) .
\end{aligned}
$$

Choose the functions $\psi_{j k}$ for $f$ as in $\S 4$ and $\tilde{\psi}_{j k}$ similarly for $g$ and set

$$
\begin{aligned}
& \eta_{f}^{*}:=\left(\frac{\left|F\left(H_{0}\right)\right|^{\gamma_{f}-\varepsilon_{1} \sigma_{n}+1}\left|F_{n_{f}}^{\prime}\right| \Pi_{j, k}\left|\psi_{j k}\right|^{\varepsilon_{1} / q}}{\Pi_{j=1}^{q}\left|F\left(H_{j}\right)\right|^{\omega_{f}^{(j)}}}\right)^{1 /\left(\sigma_{n_{f}+\varepsilon_{1} \tau_{n}}\right)}, \\
& \eta_{g}^{*}:=\left(\frac{\left|G\left(H_{0}\right)\right|^{\gamma^{-}-\varepsilon_{2} \sigma_{n} g^{+1}}\left|G_{n_{g}}\right| \Pi_{j, k}\left|\tilde{\psi}_{j k}\right|^{\varepsilon_{2} / q}}{\Pi_{J=1}^{q}\left|G\left(H_{j}\right)\right|^{\omega_{g}^{(j)}}}\right)^{1 /\left(\sigma_{n_{g}}+\varepsilon_{2} \tau_{n}\right)} .
\end{aligned}
$$

We define a new pseudo-metric by

$$
d \psi^{2}:=\frac{|\chi|^{2}}{\left|F\left(H_{0}\right)\right|^{2}\left|G\left(H_{0}\right)\right|^{2}} \eta_{f}^{*} \eta_{B}^{*}|d z|^{2} .
$$

Here, we may assume that all exponents appearing in the above are rational numbers. As is easily seen, $d \psi^{2}$ is a well-defined pseudo-metric on $\bar{M}$. On the other hand, for an arbitrary s-ple meromorphic form $\varphi$ on $M$ it holds that $\sum_{p \in \bar{M}} \nu_{d \varphi}(p)=s(2 \gamma-2)$. For a sufficiently large integer $s$, we can find an $s$-ple meromorphic form $\varphi=\varphi_{z}(d z)^{s}$ such that $d \psi^{2}=\left|\varphi_{z}\right|^{2 / s}|d z|^{2}$ for each holomorphic 
local coordinate $z$. It follows that

$$
\sum_{p \in \bar{M}} \nu_{d \psi}(p)=2 \gamma-2
$$

where $\gamma$ denotes the genus of $\bar{M}$. If we take a nonzero holomorphic function $g$ with $\nu_{g}=\min \left\{\nu_{\omega_{i}} ; 1 \leqq i \leqq m\right\}$ in a neighborhood of each $a \in \bar{M}$, we have

$$
\nu_{\omega}=\nu_{g}+\nu_{\omega / g}=\nu_{d s}+\nu\left(f, H_{0}\right)
$$

on $\bar{M}$. We define the degree of $f$ by

$$
d_{f}:=\sum_{z \in H_{0}} \nu\left(f, H_{0}\right)(z),
$$

which does not depend on the choice of the hyperplane $H_{0}$. We then have

$$
2 \gamma-2=\sum_{z \in \bar{M}} \nu_{\omega}(z)=\sum_{z \in \bar{M}} \nu_{d s}(z)+d_{f}=\sum_{l=1}^{K} \nu_{d s}\left(p_{l}\right)+d_{f},
$$

where we used the fact that $\nu_{d s} \equiv 0$ on $M$. Similarly, we get

$$
2 \gamma-2=\sum_{l=1}^{K} \nu_{d \tilde{s}}\left(a_{l}\right)+d_{g} .
$$

Comparing the definition of $d \psi^{2}$ and $d \tau^{2}$, we have

$$
\nu_{d \psi}=\nu_{d \tau}+\left(\frac{A}{2}-1\right) \nu_{F\left(H_{0}\right)}+\left(\frac{B}{2}-1\right) \nu_{G\left(H_{0}\right)}+\nu_{0},
$$

where

$$
\begin{aligned}
\nu_{0}:= & \frac{\varepsilon_{1}}{2\left(\sigma_{n_{f}}+\varepsilon \tau_{n_{f}}\right)}\left(\frac{1}{q} \sum_{j, k} \nu_{\psi_{j k}^{z}}-\sum_{0 \leqq k \leqq n_{f}} \nu_{\left|F_{k}\right|}\right) \\
& +\frac{\varepsilon_{2}}{2\left(\sigma_{n_{g}}+\varepsilon \tau_{n_{g}}\right)}\left(\frac{1}{q} \sum_{j, k} \nu_{\tilde{\phi}_{j k}^{z}}-\sum_{0 \leqq k \leqq n_{g}} \nu_{\left|G_{k}\right|}\right) \geqq 0 .
\end{aligned}
$$

Since $\nu_{d \tau} \geqq 0$ on $M$ by Proposition 3.10 , we have by the use of (6.9)

$$
\begin{aligned}
\sum_{l=1}^{K} \nu_{d \tau}\left(a_{l}\right) & =\sum_{z \in \bar{M}} \nu_{d \tau}(z)-\sum_{z \in M} \nu_{d \tau}(z) \\
& \leqq \sum_{z \in M} \nu_{d \psi}(z)-\sum_{z \in \bar{M}}\left(\left(\frac{A}{2}-1\right) \nu_{F\left(H_{0}\right)}(z)+\left(\frac{B}{2}-1\right) \nu_{G\left(H_{0}\right)}(z)\right) \\
& =2 \gamma-2-\left(\frac{A}{2}-1\right) d_{f}-\left(\frac{B}{2}-1\right) d_{g} .
\end{aligned}
$$

Since

$$
2 \gamma-2=\frac{1}{2} \sum_{l=1}^{K}\left(\nu_{d s}\left(a_{l}\right)+\nu_{d} \tilde{s}\left(a_{l}\right)\right)+\frac{d_{f}+d_{g}}{2}
$$

by $(6.10)$ and $(6.11)$, we obtain 


$$
\sum_{l=1}^{K} \nu_{d \tau}\left(a_{l}\right) \leqq \frac{1}{2}\left(\sum_{l=1}^{K} \nu_{d s}\left(a_{l}\right)+\nu_{d \tilde{s}}\left(a_{l}\right)\right)-\left(\frac{A}{2}-\frac{3}{2}\right) d_{f}-\left(\frac{B}{2}-\frac{3}{2}\right) d_{g} .
$$

On the other hand, according to Chern-Osserman's theorem ([4, Lemma 2]),

$$
\nu_{d s}\left(p_{l}\right) \leqq-2, \quad \nu_{d \tilde{s}}\left(p_{l}\right) \leqq-2 \quad(1 \leqq l \leqq K) .
$$

'This gives

$$
\sum_{l=1}^{K} \nu_{d \tau}\left(a_{l}\right) \leqq-2 K-\frac{1}{2}\left((A-3) d_{f}+(B-3) d_{g}\right) .
$$

Here, if we choose a sufficiently small positive $\varepsilon_{1}, \varepsilon_{2}$ and $\varepsilon$, then every term of the right hand side except the first may be assumed to be smaller than an arbitrarily small pre-assigned positive number. This implies that there is some $l_{0}$ with $\nu_{d \tau}\left(a_{l_{0}}\right)<-1$. It follows that

$$
\int_{M} d \Omega_{d \tau^{2}}=+\infty
$$

On the other hand, by the same argument as in the proof of Theorem 5.3 we can show $\int_{M} d \Omega_{d \tau^{2}}<\infty$ because (3.6) holds in this case too. This is a contradiction. We have Theorem 6.7.

\section{REFERENCES}

[1] L. V. Ahlfors, An extension of Schwarz's lemma, Trans. AMS., 43 (1938), 359364.

[2] W. Chen, Cartan's conjecture: Defect relation for meromorphic maps from parabolic manifold to projective space, Ph. D. dissertation, Notre Dame University, 1987.

[3] W. Chen, Defect relations for degenerate meromorphic maps, Trans, AMS, 319 (1990), 499-515.

[4] S.S. Chern and R. Osserman, Complete minimal surface in euclidean $n$-space, J. Analyse Math., 19 (1967), 15-34.

[5] H. Fujimoto, On the number of exceptional values of the Gauss map of minimal surfaces, J. Math. Soc. Japan, 40 (1988), 235-247.

[6] H. Fujimoto, Modified defect relations for the Gauss map of minimal surfaces, J. Differential Geometry, 29 (1989), 245-262.

[7] H. Fujimoto, Modified defect relations for the Gauss map of minimal surfaces, II, J. Differential Geometry, 31 (1990), 365-385.

[8] H. Fujimoto, Modified defect relations for the Gauss map of minimal surfaces, III, Nagoya Math. J., 124 (1991), 13-40.

[9] H. Fujimoto, Unicity theorems for the Gauss maps of complete minimal surfaces, J. Math. Soc. Japan, 45 (1993), 481-487.

[10] H. Fujimoto, Value distribution theory of the Gauss map of minimal surfaces in $\boldsymbol{R}^{m}$, Aspect of Math., Vol. E21, Vieweg, Wiesbaden, 1993. 
[11] R. Nevanlinna, Einige Eindeutigkeitssätze in der Theorie der meromorphen Funktionen, Acta Math., 48 (1926), 367-391.

[12] E. I. NochkA, On the theory of meromorphic functions, Soviet Math. Dokl., 27 (2) (1983), 377-381.

[13] R. Osserman, A survey of minimal surfaces, 2nd edition, Dover Publ. Inc., New York, 1986.

[14] B. V. Shabat, Distribution of values of holomorphic mappings, Transl. Math. Monographs Vol. 61, AMS, 1985.

[15] S. T. YAU, Some function-theoretic properties of complete Riemannian manifolds and their applications to geometry, Indiana Univ. Math. J., 25 (1976), 659-670.

Department of Mathematics

FACUlTy OF SCIENCE

KANAZAWA UNIVERSITY

KaKUMA-MACHI, KaNAZAWA, 920-11

JAPAN 